\title{
Anesthesia for Cerclage
}

1. Performed for cervical insufficiency (recurrent second-trimester pregnancy losses with painless cervical dilation, herniation followed by rupture of fetal membranes, and short labor with delivery of live, immature infant)

2. Can be transvaginal or transabdominal

3. Performed prophylactically (before or during pregnancy), therapeutically (when cervical changes are noted in current pregnancy) or emergently (in patients with marked cervical changes including membrane exposure to vaginal environment)

4. Uterine relaxation is essential to replace bulging fetal membranes (administer volatile anesthetic or tocolytic) to decrease the risk of membrane rupture.

5. Transvaginal cervical cerclage can be performed under spinal, epidural, or general anesthesia.

(a) The degree of cervical dilation may influence the choice of anesthesia.

(b) GA with volatile anesthetics may be needed if the cervix is dilated and uterine relaxation is needed.

(c) Sensory blockade from sacral dermatomes to T10 is necessary because both the cervix (L1 to T10) and vagina and perineum (S2-S4) require anesthesia 